\title{
Optimization of Spray Drying Parameters for Broken Rice Maltodextrin Powder and its Microencapsulation Efficiency Study on VCO Microcapsule
}

\author{
Zahiah Mohamed Amin ${ }^{1}$, Soo Peng Koh ${ }^{* 2}$, Nur Syazwani Abdul Hamid ${ }^{2}$, Chin Ping Tan ${ }^{1}$ \\ ${ }^{1}$ Department of Food Technology, Faculty of Food Science and Technology, Universiti Putra Malaysia, 43400 Serdang, \\ Selangor, Malaysia \\ ${ }^{2}$ Biotechnology \& Nanotechnology Research Center, Malaysian Agricultural Research \& Development Institute (MARDI) \\ Headquarters, Persiaran MARDI-UPM, 43400 Serdang, Selangor, Malaysia
}

Received: February 13, 2018; Accepted: March 2, 2018; Published: April 10, 2018

*Corresponding author: Soo Peng Koh, Biotechnology \& Nanotechnology Research Center, Malaysian Agricultural Research \& Development Institute (MARDI) Headquarters, Persiaran MARDI-UPM, 43400 Serdang, Selangor, Malaysia, E-mail : karenkoh@mardi.gov.my

\begin{abstract}
Maltodextrins that contain both simple sugars and polymers of saccharides are widely used in food and pharmaceutical applications as coating agent. To date, there is lack of information of maltodextrin made from Broken Rice (RB), a by-product from rice milling process. Our aim of this study was to produce the high quality of RB maltodextrin powder to be used in the food and pharmaceutical industries and its efficacy as a microencapsulating agent was determined. A RSM comprising a five-level, three-factorial, Central Composite Rotatable Design (CCRD) were used to determine the spray dryer operating parameters to obtain the optimal yield of RB maltodextrin powder. Through RSM-based optimization and verification modeling, the optimal operating parameters of the spray dryer were identified, resulting in a maximal rate of recovery of maltodextrin powder $(92.71 \% \pm 3.67)$ that was closer to the predicted value $(94.07 \%)$. In the microencapsulation study, Virgin Coconut Oil (VCO) was used as the active ingredient. RB maltodextrins with a DE value of $10-14$ exhibited a significantly higher $(P<0.01)$ Microencapsulation Efficiency (MEE) value (94.39\%) and smaller particle size $(13.38 \mu \mathrm{m})$ than the commercial $(\mathrm{COM})$ maltodextrin (81.15\%) with a particle size of $13.44 \mu \mathrm{m}$. The RB maltodextrin was compatible with the COM maltodextrin (control), which was further proven by the microstructure of the capsules viewed under a Scanning Electron Microscope (SEM).
\end{abstract}

Keywords: Broken Rice, Maltodextrin, Spray Drying, Response Surface, Microencapsulation Efficiency

\section{Introduction}

Starch is a basis of our food and industrial economy. Although starch is mainly used as food, it can be readily converted chemically and biologically into many useful and diverse products, such as paper, textiles, adhesives, beverages, confectioneries, pharmaceuticals and plastics [1]. Maltodextrin [(C6H1005)n.H2O] is a non-sweet compound, consisting of a mixture of saccharides with a molecular-weight between that of polysaccharides and oligosaccharides with a Dextrose Equivalent (DE) value less than 20, which exists as a white powder [2]. Maltodextrins with different molecular-weights can be produced from starch through either enzymatic modification using $\alpha$-amylase or acid hydrolysis [3]. The DE is a measure of the reducing sugar power of starch, expressed as the percentage of D-glucose in the dry-matter starch hydrolysate. The DE value is the inverse of the average Degree of Polymerization (DP) of anhydrous glucose units [4]. The main sources of commercially available maltodextrin are produced using potato, wheat, waxy corn and tapioca starch [5]. Maltodextrins are widely used in food and pharmaceutical applications. These applications include as a coating agent for microencapsulation in the preparation of agarose microgels for food composites in wound healing as mimetic of edible vegetable fat and in low-calorie food stuffs [6 $-10]$.

The main objective of this study was to produce the highest yield of RB maltodextrin powder using Response Surface Methodology (RSM) to optimize the parameters of the spray drying process. RSM is a valuable technique in multifactorial experiments because it is less time consuming than experimental methods and can be used to identify the truly optimal values of the factors [11]. In this study, a five-level, three-factorial, Central Composite Rotatable Design (CCRD) was used to evaluate the interactive effects amongst the three factors, which were the inlet temperature, the feed flow and the aspirator rate. Additionally, the optimization verification model was used to determine the conditions for the maximal recovery of maltodextrin powder.

VCO is well known for its high value functional oil that has received public attention due to its nutraceutical benefits. Compared with other edible oils, VCO has unique characteristics, such as crystal clarity, a pleasant odor, high resistance to rancidity, a narrow range of melting temperature, easy digestibility and absorbability [12]. However, one of the natural characteristics of VCO is that it solidifies at a temperature below $24^{\circ} \mathrm{C}$, which makes it difficult to consume and store, particularly in cold countries. Therefore, the development of microencapsulated VCO using a spray drying technique would solve this problem without changing the condition or quality of the oil. Thus, the second 
objective of this study is to study the microencapsulating effects of RB maltodextrin powder as a wall material within a Virgin Coconut Oil (VCO) model system for future food applications.

\section{Materials and Methods \\ Preparation of RB Maltodextrin}

The Broken Rice (RB) samples (blends of the local varieties MR 219 and MRR 220) were purchased from a local market (Selangor, Malaysia). RB starch was prepared at the laboratory scale according to method of Koh, et al. [13]. Low DE value RB (DE 10-14) maltodextrin was produced via enzymatic hydrolysis of RB starch using a commercial $\alpha$-amylase, BAN 480 L (Novozymes, Denmark) based on a MARDI in-house method.

\section{Experiential Design and Optimization using RSM}

A five-level, three-factorial CCRD was employed in this study. The fractional factorial design consisted of 8 factorial points, 6 axial points and 6 center points, for a total of 20 experiments. The variables and the ranges selected for achieving the maximal recovery of maltodextrin powder were as follows: inlet temperature $\left(140-170^{\circ} \mathrm{C}\right)$; feed flow $(9-15 \%)$ and aspirator rate $(85-95 \%)$. The data obtained experimentally were analyzed using Design Expert 6.06 version and were analyzed in three main steps:

- analysis of variance (ANOVA),

- performing a regression analysis and

- Plotting the response surface. The level of significance for all of the tests was set at a 95\% confidence level.

\section{Preparation of microencapsulated VCO powder}

RB maltodextrin of DE group 10-14 was supplied by MARDI (Serdang, Malaysia). Cassava (COM) maltodextrin with purity of $>90 \%$ was obtained from San Soon Seng Food Industries Sdn. Bhd. (Selangor, Malaysia) and was used as a reference because it was known to produce high quality maltodextrin. Virgin Coconut Oil (VCO) was purchased from Cocorosco Sdn. Bhd. (Johor, Malaysia). All the chemicals used for this analysis were of analytical grade. Gum arabic (food grade) was used as a stabilizer and purchased from the Markaids (M) Sdn. Bhd (Selangor, Malaysia). The emulsion of $200 \mathrm{~mL}$ was prepared at a core/wall material ratio of 1:3. A small amount of gum Arabic $(2.0 \%, \mathrm{w} / \mathrm{v})$ was added together with maltodextrin $(15.0 \%, \mathrm{w} / \mathrm{v})$ and dispersed thoroughly in deionized water with a controlled temperature of $40^{\circ} \mathrm{C}$ water bath. The VCO $(5.0 \%, \mathrm{w} / \mathrm{v})$ was added drop wise to the continuous stirring phase to form the emulsion. These coarse emulsions were homogenized in a shear homogenizer (Silverson L4R, Buckinghamshire, UK) for $1.5 \mathrm{~min}$ at 7,000 rpm to produce a finer emulsion before subjecting it to the spray drying process. The emulsions were spray dried with a mini-spray dryer (model: Büchi B-290, Büchi Labortechnik AG, Switzerland) equipped with a standard $0.7 \mathrm{~mm}$ standard diameter nozzle. The inlet and outlet temperatures of the spray dryer were maintained at $170 \pm 2^{\circ} \mathrm{C}$ and $80 \pm 2^{\circ} \mathrm{C}$, respectively.

\section{Microencapsulation efficiency (MEE) in VCO microcapsules}

The total oil contents of VCO microcapsules were determined by Lim, et al. [14] with minor modifications. Five grams of VCO microcapsules was mixed with $20 \mathrm{~mL}$ of water at $50^{\circ} \mathrm{C}$ in a 250 $\mathrm{mL}$ Erlenmeyer flask with a stopper. A total of $15 \mathrm{~mL}$ of deemulsification reagent was then added to the mixture and vortex before leaving it in a $70^{\circ} \mathrm{C}$ water bath for $6 \mathrm{~min}$. The resulting mixture was then centrifuged at $3000 \mathrm{x}$ g for $10 \mathrm{~min}$, and the total oil was collected. To prepare the de-emulsification reagent, $10 \mathrm{~g}$ of sodium salicylate and $10 \mathrm{~g}$ of sodium citrate were dissolved separately in double-distilled water, and the two solutions were mixed together with $18 \mathrm{~mL}$ of n-butanol, and brought up to 90 $\mathrm{mL}$ with doublevi-distilled water. The surface oil was measured by adding $200 \mathrm{~mL}$ of hexane to $5 \mathrm{~g}$ of VCO microcapsules and hand shaken for $1.5 \mathrm{~min}$. The solvent mixture was then passed through filter paper. The surface oil was collected after hexane evaporation. The MEE of different maltodextrin bases produced VCO microcapsules were calculated as follows:

$$
\text { The percentage of MEE }=\frac{[(\text { total oil }- \text { surface oil }) \times 100]}{\text { total oil }}
$$

\section{Particle Size Analysis}

A particle size analyzer was used to determine the particle size distribution of the VCO microcapsules. Each measurement time was set at $12 \mathrm{~s}$, and the background time was $10 \mathrm{~s}$. All the VCO microcapsules samples were sieved at size of $<100 \mu \mathrm{m}$ before being subjected to particle size determination to avoid particle agglomeration from the coverage of surface fat. The particle size distribution of VCO microcapsules was determined by using a Scirocco 2000 dry powder system provided with a Mastersizer 2000 using laser diffraction (Malvern, Worcestershire, UK). Measurements were performed in triplicate and the results were reported as the means.

\section{Scanning Electron Microscope (SEM)}

A SEM was used to examine the morphology and surface appearance of the VCO microcapsules. The VCO microcapsules samples were attached to a specimen stub with carbon paint. The coated microcapsules were examined in a Hitachi Hi-Tech FE-SEM model SU8000 Series at $15.0 \mathrm{kV}$.

\section{Statistical Analysis}

Data were statistically analyzed by a one-way analysis of variance (SPSS statistics version 16). Significant differences $(P<0.01)$ between means were determined by Duncan's multiple range test.

\section{Results and Discussion \\ Model Fitting and ANOVA}

The results of an ANOVA of the recovery rates of maltodextrin powder are shown in Table 1. An ANOVA was conducted to determine whether this polynomial model was significant. The model adequacy was evaluated from the results of an $\mathrm{F}$ test and 
the determination coefficient (R2). Some non-significant terms $(\mathrm{P}<0.05)$ were eliminated and the resulting equations were tested for adequacy and fitness using an ANOVA. The calculated $\mathrm{F}$ values for almost all of the responses were higher than the tabulated values, indicating that the model showed significant regression at a $95 \%$ confidence level. According to Table 1, model Prob > F value is 0.0051 less than 0.05 , which indicated that the model was significant.

The overall predictive capability of a model is commonly explained by the coefficient of determination (R2). The R2 value of the recovery rate of $\mathrm{RB}$ maltodextrin powder was 0.91 . This value was close to 1.0 , which means that the model equation is closer to the true behavior of the system, and it can be used for interpolation in the experimental domain [15]. The small P value (0.005) obtained using the ANOVA indicated that the reduced cubic polynomial model was highly significant and sufficient to represent the actual relationship between the response maltodextrin powder recovery rate (\%)] and the significant variables.

Table 1: ANOVA analysis and equation formula for the response surface reduced cubic model of the recovery rate of RB maltodextrin powder

Response: Total Maltodextrin Powder Yield

ANOVA for Response Surface Reduced Cubic Model

\begin{tabular}{|c|c|c|c|c|c|c|c|}
\hline Source & & $\begin{array}{l}\text { Sum of } \\
\text { squares }\end{array}$ & DF & Mean Square & F value & Prob $>$ F & \\
\hline \multirow[t]{11}{*}{ Model } & & 2295.03 & 11 & 208.64 & 7.05 & 0.051 & \\
\hline & A & 413.95 & 1 & 413.95 & 13.99 & 0.0057 & significant \\
\hline & $\mathrm{B}$ & 2.89 & 1 & 2.89 & 0.098 & 0.7628 & \\
\hline & $\mathrm{C}$ & 143.62 & 1 & 143.62 & 4.85 & 0.0587 & \\
\hline & $\mathrm{A}^{2}$ & 96.70 & 1 & 96.70 & 3.27 & 0.1082 & \\
\hline & $\mathrm{B}^{2}$ & 350.64 & 1 & 350.64 & 11.85 & 0.0088 & \\
\hline & $\mathrm{C}^{2}$ & 31.65 & 1 & 31.65 & 1.07 & 0.3312 & \\
\hline & $\mathrm{AB}$ & 12.03 & 1 & 12.03 & 0.41 & 0.5415 & \\
\hline & $\mathrm{AC}$ & 186.15 & 1 & 186.15 & 6.29 & 0.0365 & \\
\hline & $\mathrm{BC}$ & 42.37 & 1 & 42.37 & 1.43 & 0.2657 & \\
\hline & $\mathrm{ABC}$ & 453.16 & 1 & 453.16 & 15.32 & 0.0045 & \\
\hline \multirow[t]{3}{*}{ Residual } & & 236.66 & 8 & 29.58 & & & \\
\hline & Lack of Fit & 137.83 & 3 & 45.94 & 2.32 & 0.1920 & not significant \\
\hline & Pure Error & 98.83 & 5 & 19.77 & & & \\
\hline Cor Total & & 2531.69 & 19 & & & & \\
\hline
\end{tabular}

a Significant at "Prob > F" less than 0.05; $\mathrm{R}_{2}=0.91$

(Abbreviations: A: Inlet temperature; B: Feed flow; C: Aspirator rate)

Equation of total maltodextrin powder yield

\begin{tabular}{|c|c|}
\hline+4251.68441 & \\
\hline-26.72854 & *Inlet Temperature \\
\hline-519.01524 & *Feed Flow \\
\hline-42.77022 & ${ }^{*}$ Aspirator Rate \\
\hline-0.011513 & ${ }^{*}$ Inlet Temp ${ }^{2}$ \\
\hline+3.38312 & $*$ Feed Flow ${ }^{2}$ \\
\hline-0.059278 & ${ }^{*}$ Aspirator Rate ${ }^{2}$ \\
\hline+3.03775 & *Inlet Temp* Feed Flow \\
\hline+0.33708 & *Inlet Temp*Aspirator Rate \\
\hline+5.33817 & ${ }^{*}$ Feed Flow*Aspirator Rate \\
\hline-0.10920 & ${ }^{*}$ Feed Flow ${ }^{3}$ \\
\hline-0.033450 & *Inlet Temp* Feed Flow*Aspirator Rate \\
\hline
\end{tabular}




\section{Effect of the Parameters}

The inlet temperature, feed flow and aspirator rate were three most important factors affecting the recovery rate of $\mathrm{RB}$ maltodextrin powder. The 3D surface plots were developed using the fitted reduced cubic model (modified model) by holding one of the independent variables at a constant value and changing the levels of the other two variables to investigate the interaction relationship among variable factors.

Figure 1 shows the response surface plots of RB maltodextrin powder yield as a function of the inlet temperature, the feed flow and their mutual effect, with the aspirator rate set at $90 \%$. It was observed that the RB maltodextrin powder yield increased when the inlet temperature was increased from 140 to $155^{\circ} \mathrm{C}$ and that thereafter, it decreased with a dome shape observed. The inlet temperature and the feed flow showed an important interrelationship in determining the yield of RB maltodextrin powder. A higher recovery rate of RB maltodextrin powder was obtained at the higher inlet temperature when the feed flow was reduced from 15 to $12 \%$. However, the recovery rate of RB maltodextrin powder was decreased when the feed flow was set below $12 \%$ and a higher inlet temperature was required.

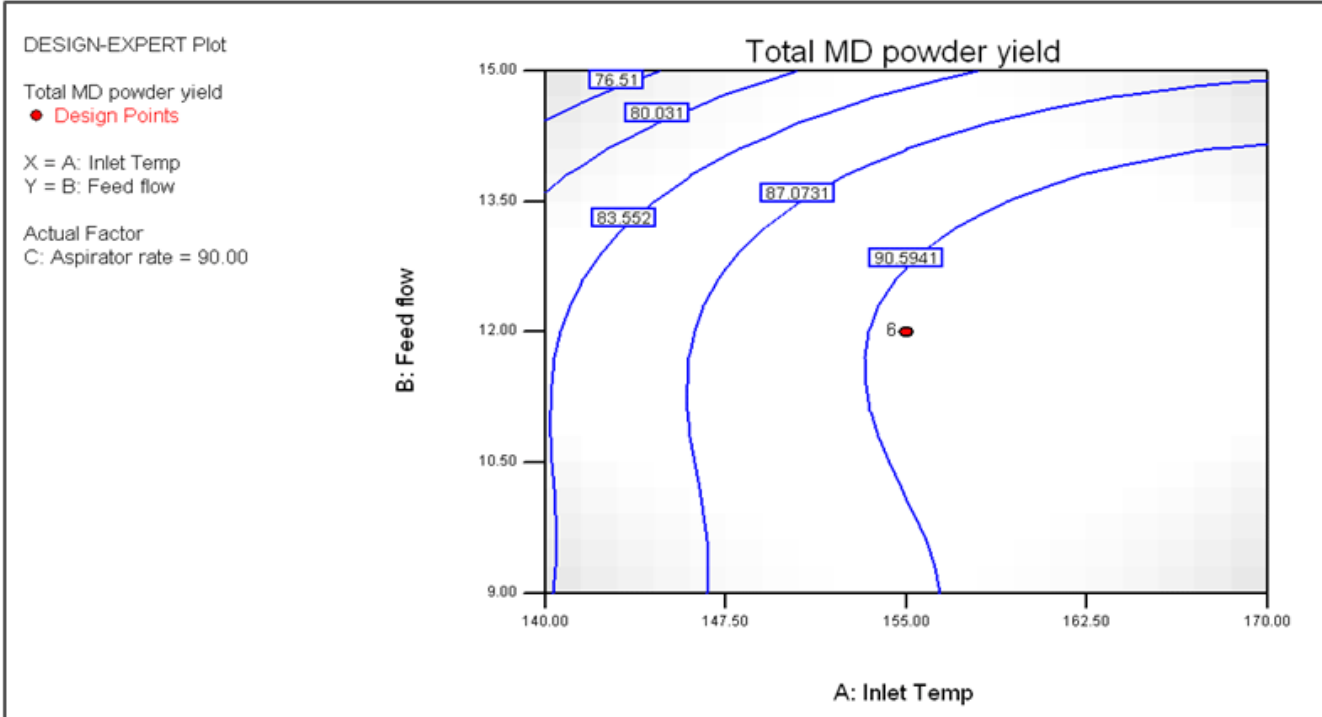

Figure 1: Response surface plots of the recovery rate of RB maltodextrin powder based the effect of the inlet temperature, the feed flow and their mutual effect, with the aspirator rate set at $90 \%$

Figure 2 shows the response surface plots as a function of the inlet temperature, the aspirator rate and their mutual effect with the feed flow constant at $9 \%$. This plot also showed a dome shape as seen in Figure 1. A higher aspirator rate was suitable for maximal recovery rate of RB maltodextrin powder only when the inlet temperature was increased from 140 to $155^{\circ} \mathrm{C}$. In this case, a lower yield of RB maltodextrin powder was observed at a lower aspirator rate, whereas a higher yield was observed at a higher aspirator rate when the feed flow was set at $9 \%$ and the inlet temperature was increased.

A higher aspirator rate was also required for the optimal recovery of RB maltodextrin with a higher feed flow when the inlet temperature was set at $155^{\circ} \mathrm{C}$, as shown in Figure 3. At a low aspirator rate, less than $90 \%$, the yield of RB maltodextrin powder decreased when the feed flow was increased to $15 \%$.

\section{Optimization and Model Verification}

The conditions for the optimal recovery of RB maltodextrin powder were predicted using the optimization function of Design Export Software. To predict the maximal percentage yield of RB maltodextrin powder in which the variables were set to the following particular values: a) the inlet temperature was set at $170^{\circ} \mathrm{C}$; b) the feed flow was set at $9 \%$, and c) the aspirator rate was set in the range of $85 \%$ and $95 \%$, the software will generate the optimal solution condition to obtain high recovery rate of RB maltodextrin powder. Based on these criteria, the optimum solution for the best recovery rate of RB maltodextrin powder was predicted with the spray dryer operating parameters was fixed at an inlet temperature of $170^{\circ} \mathrm{C}$, a feed flow of $9 \%$ and an aspirator rate of $95 \%$, which resulted in a predicted optimal recovery rate of $94.07 \%$, as shown in Table 2. The experimental value of $92.71 \%$ $(n=3)$ recovery was obtained by conducting the experiment in triplicate using the optimal operating parameters. This finding verified the high recovery rate of RB maltodextrin powder that was predicted using the RSM optimization approach. The good correlation between the actual and predicted values confirmed the validity of the response model and the optimal points obtained [16]. The interaction effects of the inlet temperature, feed flow and aspirator rate established using the RSM optimization model can be used as guidelines to predict the yield of RB maltodextrin powder without conducting experiments. 


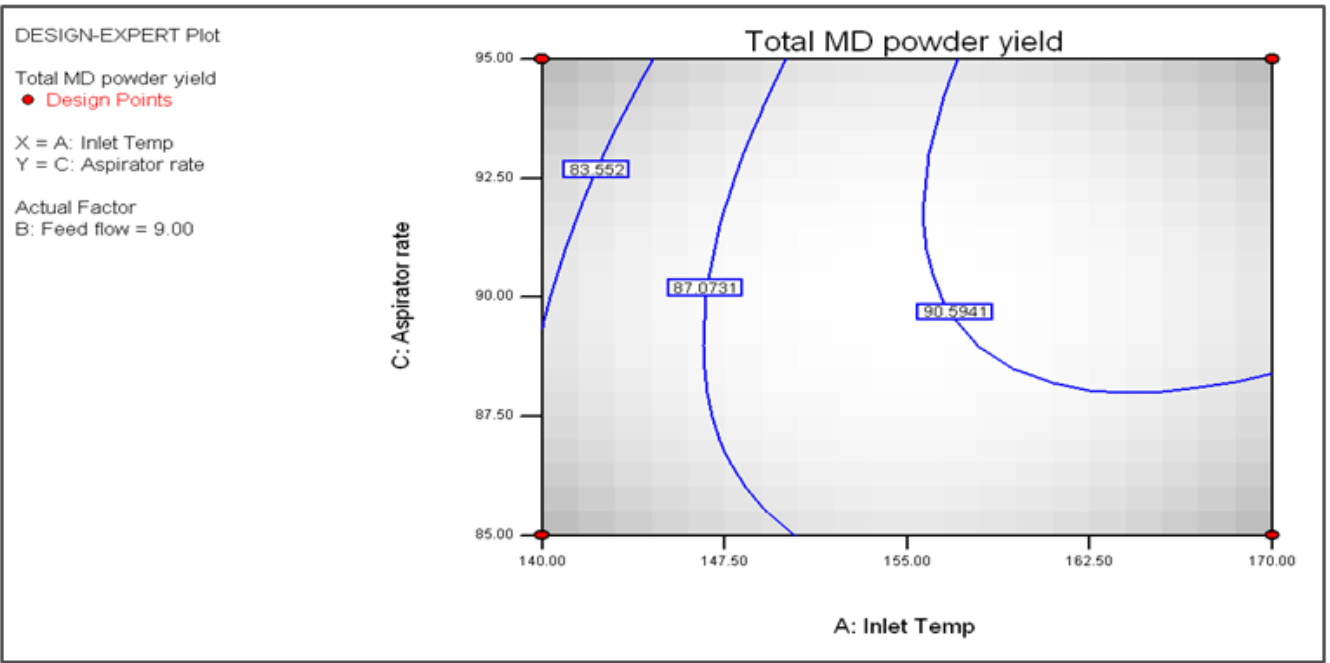

Figure 2: Response surface plots of the recovery rate of RB maltodextrin based on the effect of the inlet temperature, the aspirator rate and their mutual effect, with feed flow set at $9 \%$

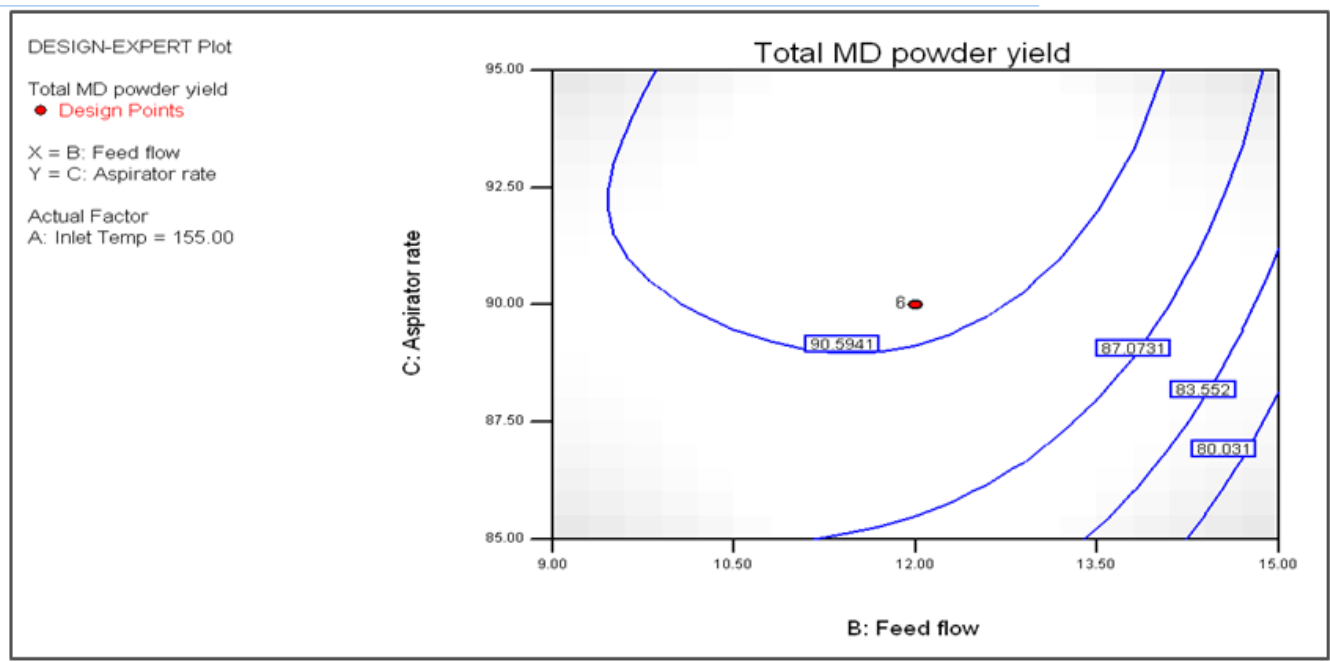

Figure 3: Response surface plots of the recovery rate of RB maltodextrin based the effect of the feed flow, the aspirator rate and their mutual effect, with the inlet temperature set at $155^{\circ} \mathrm{C}$

Table 2: Optimization and model verification of recovery rate of RB maltodextrin powder

\section{Optimization Model}

\section{$\%$ Recovery rate}

Inlet Temperature $\left({ }^{\circ} \mathrm{C}\right): 170$

Feed Flow (\%): 9

Aspirator Rate (\%): Range of 85 - 95

Recovery rate of maltodextrin powder: Maximum

Optimum solution:

Inlet temperature $\left(170^{\circ} \mathrm{C}\right)$; Feed flow (9\%) and Aspirator rate $(95 \%)$
Experimental Value

Predicted Value

$92.71 \pm 3.67(\mathrm{n}=3)$
94.07 


\section{The \% MEE and Particle Size Distribution of Microencapsulated VCO Powder}

The particle size distribution and \% MEE of microencapsulated VCO samples that were produced from different wall materials are shown in Table 3. Particle size distribution has a major role in processing, handling, shelf life and the microstructure, which is related to powder functionality, stability and flow ability [17]. Based on the results, the type of wall materials with specific DE value were shown to have a significant influence on the \% MEE of the VCO microcapsules. The RB maltodextrin exhibited a significantly higher degree $(p<0.01)$ of $\%$ MEE in comparison with COM maltodextrins. Maltodextrin with the same DE values can possess different functional and physico-chemical properties, which is dependent on the starch molecular structure itself [18] This statement was confirmed by the significant differences $(p<0.01)$ in the \% MEE values obtained for RB $(94.39 \%)$ and COM maltodextrins $(81.15 \%)$ under the same grade of maltodextrin (DE 10-14) when applied as a coating material to produce VCO microcapsules. The higher molecular-weight of maltodextrins provides an excellent encapsulation property. Based on our findings, our RB maltodextrin (DE 10-14) was able to achieve higher \% MEE than other studies which also used maltodextrin as a microencapsulating agent including $84.95 \%$ MEE, 84.25\% MEE and $81.1 \%$ MEE [20-22]. A higher percentage of MEE values indicated better protection of the core material by the wall material [14].

The particle size of microencapsulated VCO powder is presented as D4, 3 value (the volume-weighed mean or volume mean diameter) after spray drying. Wall materials based on RB maltodextrin showed a slightly smaller particle size than COM maltodextrin. Microencapsulated VCO powders coating with RB maltodextrin with DE values of 10- 14 exhibited the highest \% MEE values with particle size distribution of $13.38 \mu \mathrm{m}$. According to Ahn, et al. the particle size of the microcapsules formed had an influence on \% MEE value of encapsulated powder. The latter statement is supported by the findings of Shiga et al. (2004), the surface area of the carrier increased with an increase in the molecular-weight. This finding confirmed that the smaller particle size exhibited by RB maltodextrin resulted in an efficient and higher \% MEE (94.39\%).

Table 3: Characterization of microencapsulated VCO with different wall materials

\begin{tabular}{c|c|c|c|}
\hline $\begin{array}{c}\text { Wall } \\
\text { materials }\end{array}$ & DE value & $\begin{array}{c}\text { Powder particle } \\
\text { size } \mathbf{D}_{4,3}(\mu \mathrm{m})\end{array}$ & $\begin{array}{c}\text { MEE } \\
\mathbf{( \% )}\end{array}$ \\
\hline $\mathrm{RB}$ & $10-14$ & $13.38 \pm 0.00^{\mathrm{a}}$ & $94.39 \pm 1.40^{\mathrm{a}}$ \\
\hline $\mathrm{COM}$ & $10-14$ & $13.44 \pm 0.00^{\mathrm{a}}$ & $81.15 \pm 1.21^{\mathrm{b}}$ \\
\hline
\end{tabular}

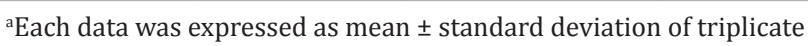
determinations. Mean values with different superscripts in the same column are significantly different $p<0.01$

(Abbreviations: RB: Broken rice Maltodextrin; COM: Commercial

Maltodextrin; DE: Dextrose Equivalents)

\section{Morphology Study of Microencapsulated VCO via SEM}

The morphological appearances of various VCO microcapsules products are presented in Figure 4. SEM was performed to assess the $\%$ MEE by determining the encapsulation ability of the wall materials as the integrity and porosity of the microcapsules [23]. This morphological analysis demonstrated a size, shape and agglomeration tendency of smaller particles between themselves and forms bigger particles. One way to reduce the agglomeration between the microcapsules is to increase the percentage of the wall material. Although the outer surfaces of VCO microcapsules exhibited irregularities (dents), they showed no pores or cracks on the VCO microcapsules. The presence of these dents has an adverse effect on the flow behavior of microcapsules; however, they did not affect the stability of the encapsulation property [24].

According to SEM observations, the surfaces of VCO microcapsule coatings with RB maltodextrin wall material exhibited the best morphology (Figure $4 \mathrm{~b}$ and c) with \% MEE of 94.4, confirming that this type of wall material has better functionality in encapsulating properties as opposed to the VCO microcapsule coatings with COM maltodextrin (Figure 4a). According to Shiga, et al. longer chains of glucose polymers (low DE group) increased the retention ability of the microcapsules [19]. This statement was further confirmed with the findings observed by SEM analysis and \% MEE value of both VCO microcapsule samples.

\section{Conclusion}

RSM comprising a five-level, three-factorial and CCRD were successfully determined the spray dryer operating parameters for the optimal yield of RB maltodextrin powder. Through RSMbased optimization and verification modeling, the optimal operating parameters of the spray dryer were identified, resulting in a maximal recovery rate of maltodextrin powder. In summary, $\mathrm{RB}$ maltodextrin exhibited a higher \% MEE value in comparison to the COM maltodextrin in the production VCO microcapsule under the same DE group. The findings indicated that RB maltodextrin has better microencapsulating properties than COM maltodextrin and were further confirmed by observing its microstructure appearance. This study provides an opportunity to create value added products and explore the potential usage of maltodextrin from RB sources for future applications in food and pharmaceuticals industries.

\section{Acknowledgments}

We gratefully appreciate the financial support of this work by Ministry of Science, Technology and Innovation of Malaysia, MOSTI (Science fund: 06-03-08-SF0320). We would like to thank the staffs of the Food Science and Technology Faculty of the University Putra Malaysia (UPM) and Malaysian Agricultural Research and Development Institute (MARDI) for their help and the use of all facilities needed to conduct this study. 


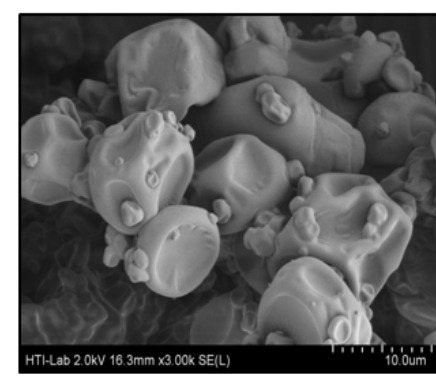

a)

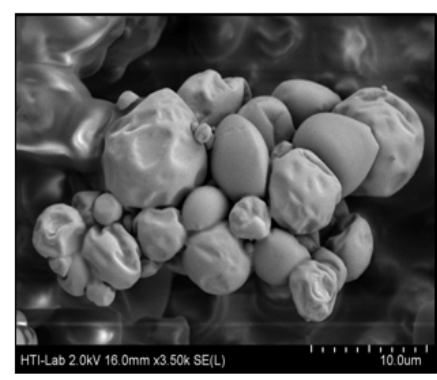

b)

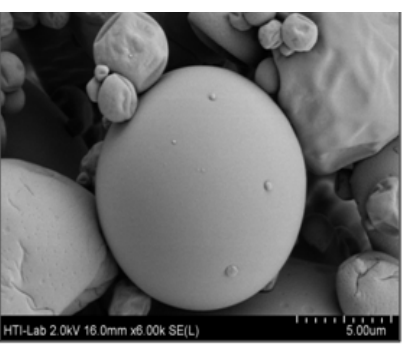

c)

Figure 4: SEM images of microencapsulated VCO with different wall materials. a) COM maltodextrin DE 10-14; b) RBmaltodextrin DE 10-14 (10 $\mu \mathrm{m})$; c) RB maltodextrin DE 10-14 (5.00 $\mu \mathrm{m})$

\section{References}

1. Nand AV, Charan RP, Rohindra D, Khurma JR. Isolation and properties of starch from some local cultivars of cassava and taro in Fiji. The South Pacific Journal of Natural and Applied Sciences. 2008;26(1):45-48.

2. Sadeghi A, Shahidi F, Mortazavi SA, Mahalati MN. Evaluation of different parameters effect on maltodextrin production by- amylase Termamyl 2-x. WASJ. 2008;3:34-39.

3. Moore GRP, Canto LRD, Amante ER, Soldi V. Cassava and corn starch in maltodextrin production. Química Nova. 2005;28(4): 596- 600.

4. Baucal DL, Dokic P, Jakovljevic J. Influence of different maltodextrins on properties of O/W emulsions. Food Hydrocolloids. 2004;18(2):233239.

5. Zielińska SJ, Fortuna T. Retrogradation of starches and maltodextrins of origin various. Acta Sci. Pol. Technol. Aliment. 2010;9: 71-81.

6. Ahn JH, Kim YP, Lee YM, Seo EM, Lee K W, Kim HS. Optimization of microencapsulation of seed oil by response surface methodology. Food Chem. 2008;107(1):98-105.

7. Loret C, Frith WJ, Fryer PJ. Mechanical and structural properties of maltodextrin/agarose microgels composites. Appl Rheol. 2007;17:31412-33136.

8. Swaim SF, Gillette RL. An update on wound medications and dressings. Compend Contin Educ. 1998; 20:1133-1144.

9. Hadnađev M, Dokić L, Hadnađev T D, Pajin B, Krstonošić V. The impact of maltodextrin-based fat mimetics on rheological and textural characteristics of edible vegetable fat. J Texture Stud. 2011;42:404-411.

10.Syed HM, Jadhav BA, Salve RV. Studies on Preparation of Low Calorie Cake using Pearl Millet (Bajra) Maltodextrin. J Food Process Technol 2011;2:125
11.Rahman RA, Illias RM, Nawawi MGM, Ismail AF, Hassan O, Kamaruddin K. Optimisation of growth medium for the production of cyclodextrin glucanotransferase from Bacillus stearothermophilus HR1 using response surface methodology. Process Biochem. 2004;39(12): 20532060.

12.Marina AM, Che Man YB, Amin I. Virgin coconut oil: emerging functional food oil. Trends Food Sci Tech. 2009; 20(10): 481-487.

13.Koh S P, Long K. Comparison of physical, chemical and functional properties of broken rice and breadfruit starches against cassava starch. J Trop Agr Food Sc. 2012;40: 211-219.

14.Lim H K, Tan C P, Bakar J, Ng SP. Effects of different wall materials on the physicochemical properties and oxidative stability of spray-dried microencapsulated red-fleshed pitaya (Hylocereuspolyrhizus) seed oil. Food Bioprocess Tech. 2012;5(4):1220-1227.

15.Baş D, Boyacı İH. Modeling and optimization I: Usability of response surface methodology. J Food Eng. 2007;78(3):836-845.

16.Koh SP, Arifin N, Tan CP, Yusoff M, Affandi S, Long K, et al. Rheological properties, oxidative stability and sensory evaluation of enzymatically synthesized medium-and long-chain triacylglycerol-based salad dressings. Eur J Lipid Sci Technol. 2008;110(12):1116-1126.

17.Phisut N. Spray drying technique of fruit juice powder: Some factors influencing the properties of product. Int Food Res J. 2012; 19:12971306.

18.Sun J, Zhao R, Zeng J, Li G, Li X. Characterization of Destrins with Different Dextrose Equivalents. Molecules. 2010; 15(8): 5162-5173.

19.Shiga H, Yoshii H, Ohe H, Yasuda M, Furuta T, Kuwahara H, et al. Encapsulation of shiitake (Lenthinus edodes) flavors by spray drying. Biosci Biotechnol Biochem. 2004;68(1): 66-71.

20.Gharsallaoui A, Roudaut G, Chambin O, Voilley A, Saurel R. Applications of spray-drying in microencapsulation of food ingredients: An overview. Food Res Int. 2007;40(9):1107-1121. 
21.Raja K, Jestin PU, Athul P V, Tamizharasi S, Sivakumar T. Formulation and evaluation of maltodextrin based proniosomal drug delivery system containing anti-diabetic (Glipizide) drug. Int J Pharm Tech Research. 2011;3: 471-477.

22.Malacrida CR, Telis VRN. Effect of different ratios of maltodextrin/ gelatin and ultrasound in the microencapsulation efficiency of turmeric oleoresin. In 11th International Congress on Engineering and Food. 2011.
23.Rosenberg M, Kopelman I J, Talmon Y. Factors affecting retention in spray-drying microencapsulation of volatile materials. J Agr Food Chem. 1990;38(5): 1288-1294.

24.Finotelli P V, Rocha-Leão MH. Microencapsulation of ascorbic acid in maltodextrin and capsul using spray-drying. In 2nd Mercosur Congress on Chemical Engineering, 4th Mercosur Congress on Process System Engineering. 2005;1-11. 